\title{
VIGILÂNCIA CLINICO-LABORATORIAL DA INFECÇÃO PELO HERPESVÍRUS HUMANO 6 (HHV-6) E HERPESVÍRUS HUMANO 7 (HHV-7) E DA CO-INFECÇÃO COM CITOMEGALOVÍRUS HUMANO (HCMV) APÓS OTRANSPLANTE HEPÁTICO
}

\author{
Clinic Laboratory Surveillance of infection by Human Herpesvírus 6 (HHV-6) and Human \\ Herpesvírus 7 (HHV-7) and Co-infection with Human Citomegalovírus (HCMV) after Liver \\ Transplantation
}

Ana Maria Sampaio, Elisabete Yoko Udo, Sohemys Silvestre Bodine, Lisandra Akemi Suzuki, Célia Regina Pavan, Raquel Bello Stucchi, Elza Cotrim Soares, Marilda Mazzali, Sandra Helena Alves Bonon, Claudio Lúcio Rossi, Sandra Cecilia Botelho Costa, Ilka de Fátima Ferreira Santana Boin

\begin{abstract}
RESUMO
Os herpesvírus humanos HHV-6 e HHV-7, após infecção primária, permanecem latentes no organismo, podendo reativar-se por período de imunossupressão. Objetivo: Realizar vigilância laboratorial da infecção por HHV-6, HHV-7 e infecção com citomegalovírus Humano (HCMV) no pós-transplante hepático, através das técnicas de antigenemia, N-PCR e sorologia. Métodos: Amostras de 32 pacientes, com idade mediana de 47 (18-66) anos, sendo 20 (62,5\%) do sexo masculino e 12 (37,5\%) do sexo feminino. A monitorização laboratorial iniciou-se no pré-operatório e, após o transplante, de modo semanal, do $1^{\circ}$ ao $2^{\circ}$ mês, quinzenal no $3^{\circ}$ mês, e mensal do $4^{\circ}$ ao $6^{\circ}$ mês. A partir de extração de linfócitos, realizou-se a detecção de HHV-6 e HHV-7, pela técnica de antigenemia. A detecção de anticorpos IgM anti HHV-6 foi realizada pelo teste Elisa. Realizou-se antigenemia, N-PCR e sorologia para HCMV, HHV-6 e HHV-7. Resultados: Com essas metodologias, a detecção de IgM anti HHV-6 foi positiva em 15,6\% dos pacientes no pré-transplante, $25 \%$ na quarta semana, $40,6 \%$ na 12a semana e em $46,9 \%$ na 24 a semana, após o enxerto. A antigenemia para HCMV, HHV- 6 e HHV7 foi positiva em $46,9 \%, 62,5 \%$ e 46,8\% respectivamente. A N-PCR para HCMV e HHV-6 ocorreu em $81 \%$ dos casos, e para HHV-7 em $46,8 \%$. Detectou-se que $50 \%$ dos pacientes estudados tiveram doença por HCMV. A doença causada pelo HHV-6 foi observada em $46,8 \%$ dos pacientes e em 15,6\% para HHV-7. A concomitância de doenças foi observada nos pacientes com HCMV e HHV-6 em $21,9 \%$ e em 15,6\% dos pacientes com HHV-6 e HHV-7. A doença causada pelos betaherpesvirus foi detectada com maior frequência ao redor da quinta semana. Os episódios de doença para HHV-6 e HHV-7 precederam ao aparecimento da doença por HCMV. Conclusão: Este estudo confirma a relevância da infecção pelo HCMV, HHV-6 e HHV-7 e a importância do monitoramento para a deteç̧ão precoce desses agentes, possibilitando a utilização de um tratamento preemptivo, com redução do risco de doença.
\end{abstract}

Descritores: Imunossupressão, Transplante de Fígado, Citomegalovírus, Herpesvirus Humano 6, Herpesvirus Humano 7; Prevalência.

Fonte Auxílio: Capes

Instituição:

Unidade de Transplante Hepático/UHT - Gastrocentro - Universidade Estadual de Campinas, Campinas/SP-Brasil

Correspondência:

Ilka de Fátima Ferreira Santana Boin

Rua Carlos Chagas 420, Cidade Universitária, CEP 13083-878, Campinas/SP, Tel: (19) 3521-8582

Email: ilkaboin@yahoo.com

Recebido em: 30/05/2012

Aceito em: 28/06/2012

\section{INTRODUÇÃO}

Com o crescimento no número de transplantes, problemas peculiares relacionados ao tratamento imunossupressivo geram importantes índices de morbimortalidade. As infecções, principalmente, causam uma série de complicações à recuperação dos pacientes transplantados. As infecções virais correspondem a uma importante fonte dessas complicações e dentre esses agentes virais, encontra-se a subfamília betaherpesvírus ( $\beta$-herpesvirinae) HCMV conhecido como o Citomegalovírus Humano, HHV-6 e HHV-7 (Herpevirus Humano Tipo 6 e Tipo 7), ${ }^{1-7}$ sendo esses três vírus pertencentes ao grupo de oito membros da família Herpesvírus de interesse 
médico. O Citomegalovírus Humano (HCMV), o Herpesvírus Humano Tipos 6 e 7 (HHV-6 e HHV-7), ambos amplamente disseminados, com soroprevalência, podendo variar de $40 \%$ a $60 \%$, em populações de alto nível sócio-econômico, enquanto em populações dos países subdesenvolvidos, a prevalência desses vírus é ainda maior, variando de $80 \%$ a $100 \%$. Esse aumento dos anticorpos deve-se aos fatores ambientais e socioeconômicos, pois são maiores nos países subdesenvolvidos. ${ }^{3-12}$ Uma vez ocorrida a primeira infecção, esses vírus permanecerão em latência por toda a vida no organismo do indivíduo. ${ }^{2,8} \mathrm{Na}$ maioria das vezes, essas infecções são assintomáticas, porém, em imunocomprometidos, como os portadores do Vírus da Imunodeficiência Adquirida (HIV) e transplantados de órgãos, essas infecções aumentam a morbidade. ${ }^{1,3}$

A transmissão dos betaherpesvírus pode ocorrer por via intrauterina, perinatal, contato direto de fluídos corporais, sexual, transfusões sanguíneas e em transplantes de órgãos, por isso, encontram-se em elevada prevalência na população em geral. ${ }^{2,8} \mathrm{O}$ risco da infecção pode estar relacionado com o status sorológico do doador e certas drogas, como a ciclosporina e azatioprina, têm sido implicadas como as facilitadoras da infecção. ${ }^{2,10-13}$ As manifestações clínicas sugestivas de doença por HCMV estão relacionadas à febre persistente, pneumonia intersticial, retinite, doença gastrintestinal e alterações laboratoriais, tais como leucopenia, trombocitopenia, atipia linfocitária e elevação das enzimas hepáticas. ${ }^{4,7}$

Episódios de convulsões, encefalites e encefalopatias têm sido associados a doenças febris causadas pelo HHV-6 e HHV-7 bem antes do exantema súbito ser, clinicamente, caracterizado no paciente. ${ }^{11}$ Os betaherpesvírus também podem predispor o receptor de transplante para infecções oportunistas. ${ }^{5}$ Nos últimos anos, HCMV, HHV-6 e HHV-7 têm sido relacionados, por vários autores, devido a sua alta prevalência na população e estar freqüentemente relacionados a grande impacto clinico após o transplante. ${ }^{14-22}$ Nesse contexto, métodos eficazes e precoces de diagnósticos laboratoriais são de grande importância no prognóstico desses pacientes. A antigenemia é considerada uma técnica para detecção precoce com alto valor preditivo para doença, sendo uma ferramenta de diagnóstico útil no pós-transplante, assim como a sorologia, e N-PCR têm sido bastante utilizados como métodos eficazes de diagnóstico para o monitoramento clinico-laboratorial.

\section{MÉTODOS}

No período de 2003 a 2006, foram monitoradas amostras de 32 pacientes de ambos os sexos que foram submetidos ao transplante de fígado na Unidade de Transplante de Fígado do Hospital de Clínicas e no Ambulatório do Gastrocentro da Universidade Estadual de Campinas (UTH - HC - Unicamp). A mediana da idade foi de $47(18-66)$ anos sendo $20(62,5 \%)$ deles pertencentes ao sexo masculino e $12(37,5 \%)$ ao sexo feminino. Para fazer parte do estudo, os pacientes deveriam ter acima de 18 anos, aceitar submeter-se às coletas de sangue no período de seis meses e regularidade para as coletas das amostras biológicas. Os que não tiveram seguimento de acordo com o protocolo não puderam fazer parte deste estudo, bem como aqueles que evoluíram ao óbito dentro do primeiro mês após o transplante.
O protocolo foi seguido de acordo com os requerimentos para pesquisas envolvendo seres humanos do Brasil e foi aprovado pelo Comitê de Ética Institucional da Faculdade de Ciências Médicas (FCM) da Universidade Estadual de Campinas Unicamp. Todos os pacientes que participaram desse trabalho foram informados sobre os procedimentos a serem adotados e concordaram com a coleta do material necessário para o estudo, mediante assinatura do Termo de Consentimento Livre e Esclarecido pelo paciente ou responsável, após todas as dúvidas terem sido esclarecidas.

A monitorização dos pacientes foi realizada prospectivamente desde o pré-transplante (imediatamente antes do ato cirúrgico - dia 0) e no pós-transplante: de modo semanal no $1^{\circ}$ e $2^{\circ}$ mês (semana 1 a 8), quinzenal no $3^{\circ}$ mês (semana 10 e 12) e mensal do $4^{\circ}$ mês até o final do $6^{\circ}$ mês (semana 16,20 e 24 ).

A imunossupressão básica consistiu de: a) inibidores de calcineurina: ciclosporina $(0,4 \mathrm{mg} / \mathrm{kg} /$ dia com nível sanguíneo variando de $150-250 \mathrm{ng} / \mathrm{ml})$ ou tacrolimus $(0,01 \mathrm{mg} / \mathrm{kg} / \mathrm{dia}$ com nível sanguíneo variando de 5-10 ng/ml); b) corticosteróides (metilprednisolna 1,0 g no intra-operatório, com redução no sétimo dia para $20 \mathrm{mg}$ até o final do primeiro mês, $15 \mathrm{mg}$ no segundo mês, $10 \mathrm{mg}$ no terceiro mês, $5 \mathrm{mg}$ no quarto mês e retirada se o paciente não fosse portador de doença autoimune); c) inibidores de purina (azatioprina na dose de $50 \mathrm{mg} /$ dia, ou micofenolato de mofetila na dose de $1000 \mathrm{mg} / \mathrm{dia} \mathrm{ou}$ micofenolato sódico na dose de $720 \mathrm{mg} / \mathrm{dia})$. A profilaxia antiviral para o Herpes simplex foi feita com aciclovir (200 $\mathrm{mg}$ de $12 / 12$ horas), por dois meses. Os pacientes não fizeram profilaxia para o HCMV, a não ser que o doador fosse soropositivo e o receptor soro-negativo. Os pacientes com doença por HCMV, com sintomas clínicos, receberam ganciclovir, por via intravenosa, na dose de $5 \mathrm{mg} / \mathrm{kg}$ duas vezes ao dia, por seis semanas, seguido por dose de manutenção.

Os critérios para caracterizar diagnóstico de infecção por HCMV foram considerados quando foram constatados dois ou mais N-PCR positivos em amostras extraídas de DNA de granulócitos de sangue periférico, consecutivos num intervalo de 30 dias segundo critérios propostos por e/ou antigenemia positiva (uma ou mais células positivas) segundo autores. ${ }^{710,14,23}$ Para HHV-6, a definição de infecção ativa foi baseada na positividade da N-PCR em DNA extraído de leucócitos totais, e/ ou antigenemia positiva (uma ou mais células positivas) segundo os relatos de literatura. ${ }^{8,9}$ E para o HHV-7 foi definida quando pelo menos uma amostra de soro resultou positiva pela N-PCR e/ou antigenemia positiva (uma ou mais células positivas). ${ }^{8,13}$

Para a caracterização de diagnóstico para a co-infecção ativa dos betaherpesvírus, considerou-se quando dois ou mais vírus foram detectados simultaneamente na mesma amostra. ${ }^{8,9}$ A doença por HCMV foi considerado além das evidências laboratoriais de infecção ativa citadas anteriormente, fez-se necessário a presença de manifestações clinicas compatíveis com aquelas sabidamente causadas pelo HCMV, como a pneumonia intersticial, doença gastrintestinal, hepatite, retinite, nefrite, miocardite, febre maior ou igual a $38^{\circ} \mathrm{C}$, no mínimo por três dias sem foco detectado, acompanhada por leucopenia $(\leq 3.000 /$ $\mathrm{mm} 3)$ e plaquetopenia $(\leq 100.000 / \mathrm{mm} 3)$ afastadas outras causas, de acordo com os critérios já descritos por outros autores.14 Para caracterizar a provável doença por HHV-6, além das evidências laboratoriais de infecção ativa citadas anteriormente, fez-se necessária a presença de manifestações clínicas compatíveis com a de HHV-6, como a febre, erupções cutâneas, disfunção hepática (alanina aminotransferase > 100IU/ml), disenteria, pneumonite intersticial, microangiopatia trombótica, encefalite e principalmente mielossupressão (sendo afastadas outras causas virais e bacterianas). ${ }^{2,14,16,17,24}$ 
Para o HHV-7 não há ainda consenso em relação às manifestações clínicas, mas, sintomas como febre, encefalite, pneumonite intersticial, hepatites, disfunção e rejeição do enxerto e alterações laboratoriais como leucopenia $(\leq 3.000 /$ $\mathrm{mm} 3)$ e plaquetopenia $(\leq 100.000 / \mathrm{mm} 3)$ e associados a AGM positiva (pelo menos uma célula) e/ou $1 \mathrm{~N}$-PCR positivo foram considerados como doença. ${ }^{13}$

Foram também avaliadas as infecções bacterianas concomitantes, definidas como aquelas que ocorreram após a cirurgia e diagnosticadas laboratorialmente. Os episódios clínicos relatados foram comparados e analisados com a presença de infecção ativa para cada um dos vírus estudados.

Devido à disfunção e à rejeição do enxerto, foi realizada avaliação da função hepática baseada na elevação sérica da alanina aminotransferase, fosfatase alcalina, gama-glutamil transpeptidase e das bilirrubinas, usando-se os padrões e protocolo da UTH-HC-Unicamp. Os episódios de rejeição do enxerto foram documentados, baseados em análises histopatológicas de material obtido por biópsia hepática. Os critérios de Banff foram utilizados para classificação e determinação do grau e tipo de rejeição celular. ${ }^{4}$

A partir de linfócitos extraídos de sangue periférico, realizou-se a detecção de antígenos de HHV-6 com anticorpos monoclonais C65206M (marca Biodesign International, France) e MAB8535 (marca Biodesign International - USA) e conjugados de soro de coelho anti-IgG de camundongo Z0412-1 (marca Dako - CA, USA) e soro de cabra anti-IgG de coelho marcado com peroxidase 81-6120 (marca Zymed, CA, USA). Para a detecção de antígenos de HHV-7 foi utilizado o anticorpo monoclonal de rato KR4 (marca Advance Biotechnologies, Canada), conjugado soro de coelho anti-imunoglobulina de camundongo P0260 (marca Dako Cytomation, USA) e conjugado soro de cabra anti-IgG de coelho 81-6120 (marca Zymed, CA, USA) diluídos em PBS/BSA e aplicados sobre a fixação celular. Foi realizada a detecção de anticorpos IgM anti HHV-6 seguindo as especificações do kit pelo teste de Elisa (marca Panbio, Austrália).

Os resultados obtidos nos procedimentos técnicos do estudo foram comparados com outros achados laboratoriais, tais como; antigenemia para HCMV, N-PCR para HCMV, HHV-6 e HHV-7 e sorologia para HCMV, bem como os achados clínicos relatados nos prontuários dos transplantados da Unidade de Transplante Hepático UTH - HC e/ou informados pela equipe médica.

Após todos os testes serem realizados, foram contabilizados para efeito de análise. Para comparação de variáveis categóricas foi utilizado o teste qui-quadrado, com uso do teste exato de Fisher quando necessário, utilizando-se o programa estatística 7.0 (CA,USA). Houve significância estatística quando o p > 0,05 .

\section{RESULTADOS}

A sorologia IgG (imunoglobulina $\mathrm{G}$ ) para $\mathrm{HCMV}$ foi positiva em $87,5 \%(28 / 32)$ dos pacientes no dia 0 do estudo. A detecção de IgM anti HHV- 6 foi positiva em $15,6 \%$ dos pacientes no prétransplante, $25 \%$ na quarta semana, $40,6 \%$ na $12^{\text {a }}$ semana e em $46,9 \%$ na $24^{\text {a }}$ semana após o enxerto (figura 1 )
Figura 1. Número de episódios de positividade dos exames de sorologia IgM (imunoglobulina M), N-PCR (reação de cadeia da polimerase) e AGM (antigenemia) para HHV-6 (herpesvirus humano tipo 6) de acordo com a semana de coleta

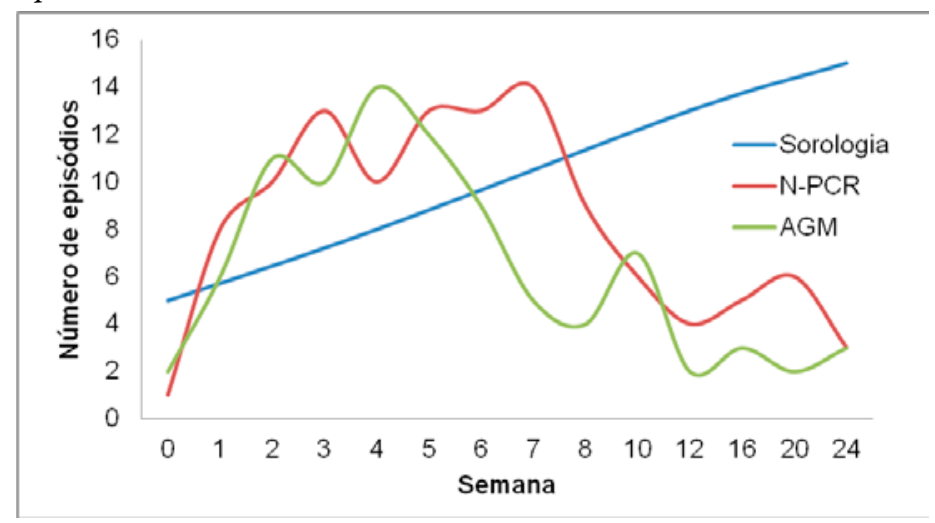

Houve 13 episódios de rejeição celular aguda em oito pacientes $(8 / 32=25 \%)$, porém somente dois $(2 / 13=15,4 \%)$ foram associados à doença HCMV e dois $(2 / 13=15,4 \%)$ à doença HHV-6. Os episódios de infecção bacteriana ocorreram em 26 $(26 / 32=81,2 \%)$, e em $13(13 / 26=50 \%)$ deles houve associação com a doença HHV-6 e HCMV.

A antigenemia para HCMV, HHV-6 e HHV-7 foi positiva em $46,9 \%, 62,5 \%, 46,8 \%$, respectivamente. A antigenemia para HCMV foi detectada em 15 casos (46,9\%), sendo que a maioria apresentou manifestações clinicas, tais como disfunções gastrintestinais acompanhadas de febre. A N- PCR para HCMV e HHV-6 detectou sequência de DNA em $81 \%$ dos casos, e para HHV-7 foi de $46,8 \%$. Detectou-se que $50 \%$ dos pacientes estudados foram diagnosticados com a doença por HCMV. A doença causada pelo HHV-6 foi observada em 46,8\% dos pacientes e em 15,6\% para HHV-7. A concomitância de doenças foi observada nos pacientes com HCMV e HHV-6 em 21,9\% casos e de 15,6\% em pacientes com HHV-6 e HHV-7; não houve concomitância para doença HCMV e HHV-7 nem para os três vírus estudados.

A doença causada pelos betaherpesvirus estudados foi detectada com maior frequência ao redor da quinta semana. $\mathrm{E}$ os episódios de doença para HHV-6 e HHV-7 surgiram antes do aparecimento da doença por HCMV.

Figura 2: Número de episódios de doença viral detectada de acordo com a semana de coleta (HCMV = citomegalovirus; $H H V-6=$ herpesvírus humano tipo $6 ; H H V-7=$ herpesvírus humano tipo 7)

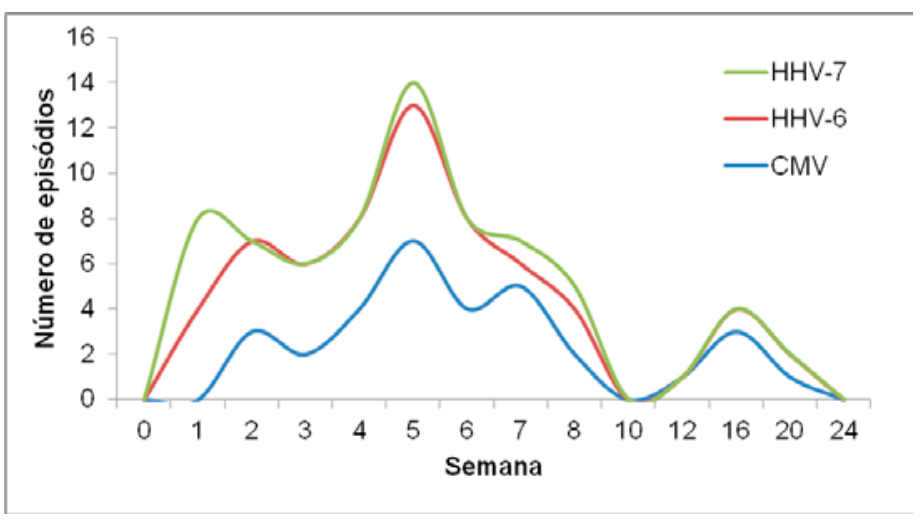




\section{DISCUSSÃO}

A infecção por HCMV após o transplante de órgãos é uma das complicações virais mais importantes que pode acometer o paciente. . $2,6,8,10,13,19,23$ Essa importância torna-se ainda mais evidente nesse contexto quando se observa que pode estar relacionada a uma miríade de complicações, como doenças oportunistas, rejeição celular aguda e crônica, infecçỗes bacterianas, dentre outras. ${ }^{18,20-32}$ Nesse estudo, investigouse a incidência das infecções pelos betaherpesvírus HCMV, HHV-6 e HHV-7 através de N-PCR, antigenemia e sorologia, e procurou-se determinar as correlações dos testes aplicados com os possíveis fatores de riscos relacionados ao impacto clínico após o transplante de fígado. Observou-se uma prevalência de $87,5 \%$ de indivíduos soropositivos para HCMV (IgG positiva), no pré-transplante, sendo esse achado compatível com a da literatura, que aponta a prevalência de infecção por HCMV em torno de $80 \%-100 \%$ da população brasileira.21 Em relação ao HHV-6 não foi possível a realização da sorologia dos respectivos doadores para determinação de infecção primária, reinfecção ou reativação. A pesquisa de anticorpos IgM para HHV-6 foi detectada em cinco pacientes $(15,6 \%)$ no pré-transplante. Consecutivamente, na quarta semana após o transplante, oito (25\%) pacientes apresentaram IgM positiva, $13(40 \%)$ na décima segunda semana e em 15 (46\%) na vigésima quarta semana após o enxerto.

Os resultados de IgM citados acima sugerem infecção primária ou uma reativação da doença após o enxerto, pois houve consecutivamente um aumento dos títulos de $\operatorname{IgM}$ no decorrer das semanas e, em muitos casos, correlações com a antigenemia ou PCRs positivos como mostra a (figura 1 ).
Na presente casuística, a deteç̧ão das infecções concentrou-se nos cinco primeiros meses de seguimento, período importante de adaptação pós-operatória em que o uso e controle da imunossupressão podem ser críticos.

A positividade para HHV-7 e HHV-6 foi detectada desde o dia 0 do estudo. Isso fez com que a ordem de reativação ou infecção nos transplantados fosse HHV-7 seguido pelo HHV-6 e, por último, o HCMV. Isso sugere que HHV-7 pode ter reativado infecções latentes por HCMV e HHV-6.

Este trabalho demonstrou correlação com dados da literatura $2,6,8,9$ e a grande importância destes agentes como causadores de morbidade em pacientes transplantados hepáticos.

\section{CONCLUSÃO}

A partir da vigilância pelas técnicas laboratoriais utilizadas nesse estudo, foi possível detectar as infecções e coinfecções pelos betaherpesvírus no pós-transplante hepático. Este estudo confirmou a relevância da infecção pelo HCMV, HHV-6 e HHV-7 nos transplantados. A realização de N-PCR e antigenemia simultaneamente são úteis para o diagnóstico desses agentes. É importante acompanhar as cargas virais após o transplante, e a antigenemia, além de ser uma técnica precoce de detecção, pode ser utilizada rotineiramente pelo beneficio de baixo custo, comparada a técnicas de moleculares. Este foi um trabalho pioneiro de antigenemia para HHV-6 e HHV-7 na equipe e demonstrou, no entanto, que outros estudos são necessários para estabelecer o uso rotineiro de antigenemia para os betaherpesvírus, para compreender melhor os aspectos clínicos que envolvem esses agentes, assim como já vem sendo realizado para o HCMV após o enxerto.

\section{ABSTRACT}

Human herpesviruses HHV-6 and HHV-7 after primary infection remain latent in the body and can reactivate after immunosuppression. Purpose: The laboratory surveillance of HHV-6 and HHV-7 and the evaluation of co-infection with HCMV human cytomegalovirus in post-liver transplantation were carried out through antigenemia, serology and N-PCR techniques. Methods: Samples from 32 patients, median age of 47 (18-66) years old, being 20 (62.5\%) male and 12 (37.5\%) were female. Biological monitoring began before transplant, weekly from 1st to 2nd months, biweekly in the 3rd month, and monthly from the 4th month to the 6th months. Extraction of lymphocytes was performed for the HHV-6 and HHV-7 antigenemia technique. Detection of IgM antibodies HHV-6 was done by the ELISA test. N-PCR and serology was applied for HCMV, HHV-6 and HHV-7. Results: With these methods, the detection of HHV-6 IgM was positive along the pre-transplant period in $15.6 \%$ of patients, $25 \%$ after the fourth week, $40.6 \%$ after the 12 th week, and $46.9 \%$ at 24 weeks after the transplant. The HCMV, HHV-6 and HHV-7 antigenemias were positive in $46.9 \%, 62.5 \%$ and $46.8 \%$ respectively, N-PCR was positive for HCMV and HHV-6 in $81 \%$ of cases, and for HHV-7 in $46.8 \%$. It was found that 50\% of patients studied had HCMV disease. The disease caused by HHV- 6 was present in $46.8 \%$ of patients and $15.6 \%$ for HHV-7. Concomitant disease was observed in patients with HCMV and HHV-6 in $21.9 \%$ and $15.6 \%$ of patients with HHV-6 and HHV-7. The disease caused by betaherpesvirus was more frequently detected around the fifth week. The HHV-6 and HHV-7 illness episodes preceded the onset of HCMV disease. Conclusion: This study confirmed the relevance of HCMV, HHV-6 and HHV-7 infections and the importance of using monitoring techniques for early detection of these agents allowing the use of a preemptive treatment with reduced risk to the disease.

Keywords: Immunosuppression; Liver transplantation; Cytomegalovirus; Human, Herpesvirus 6, Human; Human, Herpesvirus 7 , Human; Prevalence. 


\section{REFERÊNCIAS:}

1. Guardia AC, Stucchi RS, Sampaio AM, Milan A, Costa SC, Pavan $\mathrm{CR}$, et al. Human herpesvirus 6 in donor biopsies associated with the incidence of clinical cytomegalovirus disease and hepatitis $C$ virus recurrence. Int J Infect Dis. 2012 Feb;16(2):e124-9.

2. Costa FA, Soki MN, Andrade PD, Bonon SHA, Thomasini RL, Sampaio AM, et al. Simultaneous monitoring of CMV and human herpesvirus 6 infections and diseases in liver transplant patients: one-year follow-up. Clinics. 2011;66:949-53.

3. Aquino VH, Figueiredo LTM. Cytomegalovirus Infection in renal Transplant Recipients diagnosed by nested-PCR. Brazilian Journal of Medical and Biological Research. 2001;34(2):93-101.

4. Banffs Schema for Grading Lver Allograft Rejection An International Consensus Document. Hepatology. 1997;25(3):658-63.

5. Dockrell DH, Mendez JC, Jones M, Harmsen WS, Ilstrup DM, Smith TF, et al. Human herpesvirus 6 seronegativity before transplantation predicts the occurrence of fungal infection in liver transplant recipients. Transplantation. 1999;67(3):399-403.

6. Hãrma M, Hockerstedt K, Krogerus L, Lautenschlager I. Pretransplant human herpesvirus 6 infection of patients with acute liver failure is a risk factor for posttransplant human herpesvirus 6 infection of the liver. Transplantation. 2006;81:367-72.

7. Kotton CN, Humar A, Caliendo AM, Emery V, Lautenschlager I, Lazzarotto $\mathrm{T}$, et al. Transplantation Society International CMV Consensus Group. International consensus gudekines on the management of cytomegalovirus in solid organ transplantation. Transplantation. 2010;89:779-95.

8. Lautenschlager J, Lappalainen M, Linnavuori K, Suni J, Hockerstedt $\mathrm{K}$. CMV infection is usually associated with concurrent HHV-6 and HHV-7 antigenemia in liver transplant Patients. J Clin Virol. 2002;25:57-61.

9. Lautenschlager I, Halme L, Hockerstedt K, Krogerus L, Taskinen E. Cytomegalovirus infection of the liver transplant: virological, histological, immunological, and clinical observations. Transpl Infect Dis. 2006;8:21-30.

10. Milan A, Sampaio AM, Guardia AC, Pavan CR, Andrade PD, Bonon SH, et al. Monitoring and detection of cytomegalovirus in liver transplant recipients. Transplant Proc. 2011;43:1360-1.

11. Razonable, RR, Paya C. The impact of HHV-6 and 7 infection on the outcome of liver transplantation. Liver Transplantation . 2002;(8):651-8.

12. Maya TC; Azulay, DR. Infecção pelo Citomegalovirus. In: Lupi O; Silva AS; Pereira Jr. Herpes - Clinica, Diagnóstico e Tratamento, $1^{\circ}$ Ed., Medsi Editora Médica e Científica. 2000; 8:135-56.

13. Thomasini RL, Sampaio AM, Bonon SH, Boin IF, Leonardi LS, Leonardi $\mathrm{M}$, et al. Detection and monitoring of human herpesvirus 7 in adult liver transplant patients: impact on clinical course and association with cytomegalovirus. Transplant Proc. 2007;39:1537-9.

14. Ljungman P, Griffiths P, Paya C. Definition of cytomegalovirus infection and disease in transplant recipients. Clin Infect Dis. 2002;34:1094.

15. Lee SO, Brown RA, Razonable RR. Clinical significance of pretransplant choro mosomally integrated human herpesvírus- 6 in liver transplant recipients. Transplantion. 2011;92:224-9.
16. Sashihara J, Tanaka-Taya K, Tanaka S, Amo K, Miyagawa H, Hosoi $\mathrm{G}$, et al. herpesvirus 6 infection with a high viral load in cord blood stem cell transplant recipients. Blood. 2002;100:2005-11

17. Singh N, Carrigan DR. Human herpesvirus- 6 in transplantation: an emerging pathogen. Ann Intern Med. 1996 Jun 15;124(12):1065-71.

18. Seehofer, D. et al. CMV hepatitis after liver transplantation: incidence, clinical course, and long-term follow-up. Liver Transplantation. 2002;8(12):1138-46

19. Scott GM, Naing Z, Pavlovic J, Wasenko JM, Angus P, Jones R, et al. Viral factors influencing the outcome of human cytomegalovirus infection in liver transplant recipients. J Clin Virol. 2011;51:229-33.

20. Lianghui G, Shusen Z, Tinoba L, Yan S, Welling W, Anwei L. Deferred versus prophylactic therapy with gancyclovir for cytomegalovirus in allograft liver transplantation. Transplantation Proceedings . 2004;36:1502-5

21. Costa SCB. Infecção por citomegalovirus (CMV); epidemiologia, diagnóstico e tratamento. Revista Brasileira de Clínica Terapêutica n. 25 1. 1999. p 18-28.

22. Biganzoli P, Ferreyra L, Sicilia P, Carabajal C, Frattari S, Littvik A, et al. IgG Subclasses and DNA Detection of HHV-6 and HHV-7 in Healthy Individuals. J Med Virol. 2010;82:1679-83.

23. Bonon SHA, Menoni SMF, Rossi CL, Souza CA, Costa SCB. Surveillance of cytoemaglovirus infection in haematopoietic stem cell transplantation patients. J Infection. 2005;50:130-13.

24. Boutolleau DD, Cointe A, Gautheret-Dejean M, Mace H, Agut L, Grangeot-Keros, et al. No evidence for a major risk of roseolovirus vertical transmission during pregnancy. Clin. Infect. 2003;36:1634-5.

25. Almeida LNB, Azevedo RS, Amaku M, Massad E. Cytomegalovirus seroepidemiology in an urban community of São Paulo, Brazil. Rev Saúde Pública. 2001;35(2):124-9.

26. Feldstein AE, Razonable RR, Boyce TC, Freese, DK, El-Youssef $\mathrm{M}$, Perrault J, et al. Prevalence and clinical significance of human herpesviruses 6 and 7 active infection in pediatric liver transplant patients. Pediatr Transplant. 2003;7:125-9.

27. Ferreira W, Souza J. Microbiologia - volume III. Lidel - edições técnicas, lda; Lisboa, Portugal 2002

28. Bresnahan WA, Shenk T. A Subset of Viral Transcripts Packege Within Human Cytomegalovirus Particles. Science. 2000 Jun 30;288(5475):2373-6.

29. Humar A, Malkan G, Moussa G, Greig P, Levy G, Mazzulli T. Human herpesvirus- 6 is associated with cytomegalovirus reactivation in liver transplant recipients. J Infect Dis. 2000;181:1450-3.

30. Linares L, Sanclemente G, Cervera C, Hoyo I, Cofán F, Ricart MJ, et al. Influence of Cytomegalovirus Disease in Outcome of Solid Organ Transplat Patients. Transplantation proceedings. 2011;43:2145-8.

31. Scott GM, Naing Z, Pavlovic J, Wasenko, JM, Angus P, Jones R, et al. Viral factors influencing the outcome of human cytomegalovirus infection in liver transplant recipients. J Clin Virol. 2011;51:229-33.

32. Pannuti CS, Vilas B, Neto, LSVA, Ângelo, MJO, Sabbada E, Detecção de anticorpos IgM nas interações primárias e secundárias pelo citomegalovirus em pacientes submetidos a transplante renal. Ver Inst Méd Trop. 1987;29:317-22. 